Relations industrielles

Industrial Relations

\title{
La procédure d'arbitrage
}

Son usage dans la province de Québec

Volume 4, numéro 8, avril 1949

URI : https://id.erudit.org/iderudit/1023517ar

DOI : https://doi.org/10.7202/1023517ar

Aller au sommaire du numéro

Éditeur(s)

Département des relations industrielles de l’Université Laval

ISSN

0034-379X (imprimé)

1703-8138 (numérique)

Découvrir la revue

Citer cet article

(1949). La procédure d'arbitrage : son usage dans la province de Québec.

Relations industrielles / Industrial Relations, 4(8), 79-80.

https://doi.org/10.7202/1023517ar

Tous droits réservés (C Département des relations industrielles de l’Université Laval, 1949
Ce document est protégé par la loi sur le droit d'auteur. L'utilisation des services d'Érudit (y compris la reproduction) est assujettie à sa politique d'utilisation que vous pouvez consulter en ligne.

https://apropos.erudit.org/fr/usagers/politique-dutilisation/ 
entre le capital et le travail, si l'économique et le social sont placés, par l'appareil juridique qui les encadre, dans l'impossibilité de se rencontrer et de se compénétrer.

A sa base même, la réforme de l'entreprise accroche le problème de la propriété. Il est, à ce sujet, étonnant de remarquer combien habile fut, depuis un siècle, le régime capitaliste, à se faire passer comme le défenseur incontesté, l'unique gardien de la propriété personnelle, combien il fut habile, loup revêtant la peau de l'agneau, à se confondre avec elle, à se faire pur, si l'on peut ainsi s'exprimer, alors qu'en réalité, dans quatre-vingt-dix pour cent des grandes organisations commerciales et industrielles, il représentent ce qui peut exister de plus anonyme, de plus collectif, de plus diffus.

Dans un chapitre intitulé «Propriété et entreprise», M. l'abbé Bolté définit la propriété, énumère ses titres, fait ensuite les applications qui s'imposent à l'entreprise dont les modalités peuvent varier de la propriété personnelle à un rassemblement de capitaux venant des cinq parties du monde. L'auteur conclut de tout ceci, que, d'une part «sous l'inspiration du libéralisme économique, l'argent règne en maître, qu'on délaisse la fonction sociale inhérente à la propriété, le service inhérent à l'autorité », et que, d'autre part, si l'on veut corriger cet état de choses, faire de la grande entreprise \&une réalité communautaire », il faut tempérer le contrat de salariat par des éléments empruntés au contrat de société.

De son côté, M. Marcel Clément, après avoir opposé la libre entreprise à l'entreprise libérale, fait les distinctions nécessaires entre la paternité, le patronat et le paternalisme, analyse la structure de l'entreprise moderne, explique quelques-unes des solutions qui peuvent s'appliquer pour corriger ses défauts et combler ses lacunes. M. l'abbé Gérard Dion étudie la moralité de la participation aux bénéfices tandis que $M$. Clément laisse entrevoir le rôle que doit jouer le syndicalisme dans l'élaboration des réformes de structure.

Tous ceux qui veulent réellement et sincèrement travailler à la restauration de l'ordre social trouveront porfit à lire et à étudier la brochure « Réformes de structure dans l'entreprise ». Même si elle ne vide pas la question, comme le font remarquer ses auteurs, elle contribuera singulièrement à renseigner, à faire réfléchir et à ouvrir des horizons nouveaux.

(L'Action Catholique)

André Roy

\section{LA PROCÉDURE D'ARBITRAGE \\ Son usage dans la province de Québec}

On consultera avec beaucoup d'intérêt le rapport annuel du ministère du Travail pour l'année 1947-48. Des statistiques ont été compilées qui permettent au lecteur de se rendre compte de l'immense effort de conciliation et d'arbitrage qui a été donné sans bruit ni grande publicité par les conciliateurs et les arbitres désignés par l'autorité publique.

Et d'abord, un coup d'oeil rapide sur le rapport de la Commission de relations ouvrières nous indique qu'au cours de cette année les syndiqués ont déposé 676 conventions collectives couvrant 158,317 salariés. Un rapport complémentaire indique qu'en tenant compte des conventions terminées, dénoncées et non renouvelées, on constate que 1,136 conventions sont en vigueur couvrant 163,548 salariés.

Nous ne sommes pas en mesure d'expliquer le décalage considérable entre le nombre des conventions déposées au cours de l'année et celui des conventions actuellement en vigueur comme il nous est impossible d'expliquer la faible différence entre 158,317 salariés couverts par les conventions de l'année actuelle et 163,548 salariés couverts par les conventions actuellement en vigueur. A tout événement, on peut trouver ces chiffres à la page $33 \mathrm{du}$ rapport annuel.
Le tableau 9, aux pages 34 et suivantes, classe par affiliation syndicale le nombre de conventions déposées ainsi que les employés couverts durant la même année.

La Confédération des Travailleurs catholiques du Canada, inc., a à son crédit 163 conventions couvrant 44,057 salariés. 124 de ces conventions ont été conclues à la suite de négociations directes, ce qui constitue un très fort pourcentage. Par contre 39 sont passées par le stade de la conciliation et 13 par celui de l'arbitrage.

Les syndicats indépendants incorporés ou non ont à leur crédit 73 conventions couvrant 27,175 salariés; 66 de ces conventions ont été conclues par négociations directes, 7 l'ont été au stade de la conciliation et 3 seulement à celui de l'arbitrage.

Quant au Congrès canadien du Travail il compte 71 conventions intéressant 12,873 salariés. De ces conventions, 50 ont été négociées directement par les intéressés, 20 ont subi le stade de la conciliation et 14 celui de l'arbitrage.

Enfin, le Congrès des Métiers et du Travail auquel sont affiliées les unions de la Fédération américaine du Travail, a à son crédit 137 conventions visant 40,280 salaries. De ces conventions, 110 ont été signées à la suite de négociations di- 
rectes; 25 ont été soumises à la conciliation et 15 à l'arbitrage.

Nous sommes un peu surpris de constater qu'en totalisant les arbitrages indiqués dans le rapport statistique des conventions collectives de travail de la Commission de relations ouvrières (page 33) on arrive à compter 79 tribunaux d'arbitrage; or, si l'on réfère au rapport de monsieur Cyprien Miron, directeur du Service de conciliation et d'arbitrage, (page 62) on trouve que 99 tribunaux d'arbitrage ont été constitués durant l'année fiscale 1947-48. Comment expliquer la différence entre le total de $\mathbf{7 9}$ arbitrages indiqués par la Commission de relations ouvrières et celui de 99 arbitrages précisés par le Service de conciliation et d'arbitrage ?

Admettons que certains tribunaux avaient déjà commencé d'opérer avant l'année fiscale et que d'autres tribunaux n'avaient pas rendu leur sentence au ler avril, 1948. Il reste quand même qu'un décalage du quart nous apparaît exorbitant et nous inviterions les intéressés à comparer leur rapport annuel ou à donner des explications qui permettent de comprendre les écarts signalés.

A tout hasard il est intéressant de savoir par le rapport de monsieur Miron que sur les 99 arbitrages enregistrés au cours de l'année 1947-48, 34 doivent être mis au crédit de la Confédération des Travailleurs catholiques du Canada, inc., 30 à celui du Congrès des Métiers et du Travail (F.A.T.), 22 à celui du Congrès canadien du Travail (C.I.O) et 13 à des groupements indépendants.

Ce n'est certainement pas la même proportion que lon peut constater dans le rapport statistique de la Commission de relations ouvrières (page 33).

Il est possible que toutes ces différences ennuyeuses pour le public qui compulse les documents officiels soient attribuables au fait que certains arbitrages organisés par le Service ministériel affectaient des conventions collectives susceptibles d'extension aux tiers ou le règlement de griefs. Nous insistons cependant pour que des notes explicatives soient données dans les rapports officiels de façon qu'il n'y ait aucune contradiction entre les chiffres fournis par les divers départements.

Le chapitre des conflits survenus au cours de l'année 1947-48 ne manque pas non plus d'intérêt. On sera surpris d'apprendre que 80 grèves ont été déclarées (cf. pages 94 et suivantes). Sur ce nombre, 27 sont attribuables aux unions affiliées à la Confédération des Travailleurs catholiques du Canada, inc., 16 à celles affiliées au Congrès des Métiers et du Travail (F.A.T.) et 37 à celles affiliées au Congrès canadien du Travail (C.I.O.)

La plupart des grèves du C.I.O. ont eu lieu dans lindustrie du cuir et de la chaussure. Le C.I.O. a fait une grosse campagne pour s'emparer de lindustrie de la chaussure et de celle de la sacoche. Cette campagne ne semble pas avoir donné de grands résultats. Les autres grèves du C.I.O. l'ont été dans les salaisons.

Nous invitons le lecteur à se procurer ce rapport intéressant en s'adressant au Service des publications du ministère du Travail, 490, Boulevard Charest, Québec. On verra des annotations intéressantes sur la nature du conflit, sa durée, le nombre de jours ouvrables perdus et les méthodes de règlement. A noter que la conciliation post-arbitrale semble donner d'excellents succès. Nous ne sommes pas en mesure de dire si toutes les procédures prévues par la loi ont été suivies par les patrons et ies ouvriers; il serait intéressant que le rapport indiquât si ces procédures ont été violées ou non.

Somme toute la procédure de conciliation et d'arbitrage donne dans la province de Québec comme ailleurs, quand on sait y recourir, des fruits profitables. Comme on le disait récemment, sur à peu près 1,200 conventions négociées au cours d'une année les trois-quarts, soit 900 , le sont directement par les parties laissant aux conciliateurs le soin d'aider au règlement de 300 litiges.

Ces conciliations subissent parfois des échecs et nécessitent dans près d'un tiers des cas la formation d'un tribunal d'arbitrage. La majorité des sentences arbitrales sont agréées par les parties ne laissant ainsi qu'un résidu de cas malheureux où les parties doivent recourir à la force économique pour obtenir la justice. 